\title{
Spin superradiance versus atomic superradiance
}

\author{
V.I. Yukalov \\ Institut für Theoretische Physik, \\ Freie Universität Berlin, Arnimallee 14, D-14195 Berlin, Germany \\ and \\ Bogolubov Laboratory of Theoretical Physics, \\ Joint Institute for Nuclear Research, Dubna 141980, Russia
}

\begin{abstract}
A comparative analysis is given of spin superradiance and atomic superradiance. Their similarities and distinctions are emphasized. It is shown that, despite a close analogy, these phenomena are fundamentally different. In atomic systems, superradiance is a self-organized process, in which both the initial cause, being spontaneous emission, as well as the collectivizing mechanism of their interactions through the common radiation field, are of the same physical nature. Contrary to this, in actual spin systems with dipole interactions, the latter are the major reason for spin motion. Electromagnetic spin interactions through radiation are negligible and can never produce collective effects. The possibility of realizing superradiance in molecular magnets by coupling them to a resonant circuit is discussed.
\end{abstract}

Key words: spin superradiance; atomic superradiance; cooperative phenomena; optical coherence; molecular magnets

PACS: 76.20.+q, 75.45.+j, 42.50.Fx, 42.25.Kb, 07.57.Hm 


\section{Introduction}

Spin superradiance is a phenomenon that got its name because of its close analogy with superradiance occurring in atomic systems [1]. In the latter, superradiance was predicted by Dicke [2] in 1954 and nowadays it is well studied both theoretically and experimentally, being now a part of textbooks [3,4]. Spin superradiance is a relatively novel notion, being discovered experimentally $[5,6]$ in 1988, and whose exhaustive microscopic theory has been elaborated only recently (see reviews $[1,7]$ ).

Despite a similarity between atomic and spin superradiance, these phenomena are not identical, and even more, there is a fundamental distinction between them. This principal distinction is in the mechanism that triggers the initial motion and in the forces which make the system dynamics coherent [8-12]. Because of this radical difference, spin superradiance is not identical to Dicke atomic superradiance. This crucial fact is often overlooked, and it is possible to meet in literature wrong speculations caused by misunderstanding of the essentially different nature of these phenomena. A glowing example of such a confusion is comment [13] containing several incorrect statements based on the delusion that there is no any difference between atomic and spin superradiance.

The aim of the present communication is to explain the difference between the two types of superradiance and to emphasize that what is feasible for atomic systems is impossible for spin systems. A correct understanding of these points is vitally important for the proper interpolation of experiments with molecular magnets. Superradiance in these materials is principally unachievable in the same setup as for atomic systems. However, it can be realized in a different way, typical of spin systems, as has been suggested in Ref. $[14]$.

It is also crucially important to be precise in terminology. One sometimes misuses the notion of superradiance applying it vagely to any coherent radiation. This is certainly impermissible, since without a precise fixed terminology any scientific descriptions and discussions become pointless. Therefore in the present paper the notion of superradiance is used in its strictly defined and commonly accepted sense, be it atomic [2-4] or spin [1,7] superradiance: Superradiance is a self-organized collective emission of electromagnetic radiation.

This phenomenon should not be confused with spin induction or maser generation, as is explained in reviews $[1,7]$. For instance, when the spin motion is pushed by a strong transverse field, one has spin induction, which, though being a coherent process, has nothing to do with superradiance. Or, if a coherent process occurs on the temporal scale of the dephasing time $T_{2}$, this again is not related to superradiance, since it does not involve collective effects.

\section{Atomic superradiance}

The microscopic description of atomic superradiance is based on the Hamiltonian

$$
\hat{H}=\hat{H}_{a}+\hat{H}_{f}+\hat{H}_{a f},
$$


containing the terms corresponding to atoms, $\hat{H}_{a}$, field $\hat{H}_{f}$, and atom-field interactions, $\hat{H}_{a f}$. The Hamiltonian of $N$ resonant atoms is

$$
\hat{H}_{a}=\sum_{i=1}^{N} \omega_{0}\left(\frac{1}{2}+S_{i}^{z}\right)
$$

where $\omega_{0}$ is a transition frequency, $S_{i}^{z}$ is a preudospin operator characterizing the population difference, and we set $\hbar \equiv 1$. In the field Hamiltonian

$$
\hat{H}_{f}=\frac{1}{8 \pi} \int\left(\mathbf{E}^{2}+\mathbf{H}^{2}\right) d \mathbf{r},
$$

$\mathbf{E}$ is electric field and $\mathbf{H}$ is magnetic field. The latter is defined through the vector potential $\mathbf{A}$ as $\mathbf{H}=\nabla \times \mathbf{A}$. For the vector potential, the Coulomb calibration $\nabla \cdot \mathbf{A}=0$ will be used. Atom-field interactions are described by the Hamiltonian

$$
\hat{H}_{a f}=-\sum_{i=1}^{N}\left(\frac{1}{c} \mathbf{J}_{i} \cdot \mathbf{A}_{i}+\mathbf{P}_{i} \cdot \mathbf{E}_{0 i}\right),
$$

in which $\mathbf{A}_{i} \equiv \mathbf{A}\left(\mathbf{r}_{i}, t\right), \mathbf{E}_{0 i} \equiv \mathbf{E}_{0}\left(\mathbf{r}_{i}, t\right)$ is an external field, if any, the transition current is

$$
\mathbf{J}_{i}=i \omega_{0}\left(\mathbf{d} S_{i}^{+}-\mathbf{d}^{*} S_{i}^{-}\right)
$$

where $\mathbf{d}$ is a transition dipole, and the transition polarization is

$$
\mathbf{P}_{i}=\mathbf{d} S_{i}^{+}+\mathbf{d}^{*} S_{i}^{-}
$$

with $S_{i}^{ \pm}$being the ladder operators. For the pseudospin operators, the notation $S_{i}^{\alpha} \equiv$ $S^{\alpha}\left(\mathbf{r}_{i}, t\right)$ is used. Here the electric dipole transitions are assumed, although practically the same consideration is valid for magnetic dipole transitions [15].

For the best comparison of atomic coherent effects with their spin counterpart, it is necessary to represent the atomic evolution equations in terms of the pseudospin operators. This can be done by the method of elimination of field variables [16], which allows for the reduction of the equations of motion to the Heisenberg equations for the pseudospin variables. Averaging these equations over the spin degrees of freedom, we come to the equations for the transverse function

$$
u(\mathbf{r}, t) \equiv 2<S^{-}(\mathbf{r}, t)>
$$

coherence intensity

$$
w(\mathbf{r}, t) \equiv 4<S^{+}(\mathbf{r}, t) S^{-}(\mathbf{r}+0, t)>,
$$

and the average population difference

$$
s(\mathbf{r}, t) \equiv 2<S^{z}(\mathbf{r}, t)>
$$

To write down the evolution equations in a compact form, it is convenient to introduce the notation for the effective force

$$
f(\mathbf{r}, t)=f_{0}(\mathbf{r}, t)+f_{r a d}(\mathbf{r}, t)+\xi(\mathbf{r}, t)
$$


in which the first term

$$
f_{0}(\mathbf{r}, t) \equiv-2 i \mathbf{d} \cdot \mathbf{E}_{0}(\mathbf{r}, t)
$$

describes the action of an external field, the second term

$$
f_{\text {rad }}(\mathbf{r}, t) \equiv 2 k_{0}<\mathbf{d} \cdot \mathbf{A}_{\text {rad }}(\mathbf{r}, t)>
$$

corresponds to the interaction through resonant radiation with a frequency $\omega_{0}$ and wave vector $k_{0}=\omega_{0} / c$, and the last term

$$
\xi(\mathbf{r}, t) \equiv 2 k_{0} \mathbf{d} \cdot\left(\mathbf{A}_{v a c}+\mathbf{A}_{d i p}\right)
$$

is due to vacuum fluctuations and to effective transition-dipole interactions. The radiation force (12) can be represented as

$$
f_{\text {rad }}(\mathbf{r}, t)=-i \gamma_{0} \rho \int\left[G\left(\mathbf{r}-\mathbf{r}^{\prime}, t\right) u\left(\mathbf{r}^{\prime}, t\right)-\frac{\mathbf{d}^{2}}{|\mathbf{d}|^{2}} G^{*}\left(\mathbf{r}-\mathbf{r}^{\prime}, t\right) u^{*}\left(\mathbf{r}^{\prime}, t\right)\right] d \mathbf{r}^{\prime},
$$

where $\rho \equiv N / V$ is atomic density, the transfer function is

$$
G(\mathbf{r}, t)=\frac{\exp \left(i k_{0} r\right)}{k_{0} r} \Theta(c t-r),
$$

with $\Theta(\cdot)$ being the unit-step function, and where the natural width

$$
\gamma_{0} \equiv \frac{2}{3}|\mathbf{d}|^{2} k_{0}^{3}
$$

has entered the equations. Then the evolution equations for functions (7) to (9) take the form

$$
\begin{gathered}
\frac{\partial u}{\partial t}=-i\left(\omega_{0}-i \gamma_{2}-i \gamma_{2}^{*}\right) u+f s, \quad \frac{\partial w}{\partial t}=-2\left(\gamma_{2}+\gamma_{2}^{*}\right) w+\left(u^{*} f+f^{*} u\right) s, \\
\frac{\partial s}{\partial t}=-\frac{1}{2}\left(u^{*} f+f^{*} u\right)-\gamma_{1}(s-\zeta),
\end{gathered}
$$

where $\gamma_{2} \approx \gamma_{0}$ is a transverse homogeneous relaxation rate, $\gamma_{2}^{*}$ is inhomogeneous broadening, $\gamma_{1}$ is a longitudinal relaxation rate, and $\zeta$ is a stationary population difference for an atom. All details of deriving Eqs. (16) can be found in review [16].

A necessary condition for the appearance of coherent effects is the existence of strongly correlated regions, so called wave packets, of a characteristic size $L_{c}$, such that

$$
k_{0} L_{c} \ll 1 \quad\left(k_{0} \equiv \frac{\omega_{0}}{c}\right) .
$$

Coherence can arise only inside such a well correlated region. The characteristic length $L_{c}$ must be much larger than the mean interatomic distance, however it can be much smaller than the system length $L$. It is solely in the extreme case of the so-called concentrated system, for which the radiation wavelength $\lambda \gg L$, when $L_{c}=L$. But the appearance of superradiance, of course, does not require that $\lambda$ be larger than the system length. Moreover, in standard optical systems $\lambda$ is always shorter than $L$. And optical superradiance 
does exist in such systems with $\lambda \ll L$, which is, actually, a well known fact expounded in textbooks [3,4] and noticed yet in the original paper by Dicke [2] (see also discussion in Ref. [17]). When $\lambda \ll L$, then the sample becomes separated into correlated regions with $L_{c} \ll L$, and inside each of such regions coherence develops owing to condition (17). The correlation between different wave packets is weak, because of which the correlated regions radiate almost independently, thus, producing oscillations in the superradiant pulse. In lasers with large aperture, coherent radiation is always realized in the form of a bunch of filaments [18-21]. Superradiance with $\lambda \ll L$ can arise even in photonic bound-gap materials, where atomic spontaneous emission is suppressed [22]. Hence, one should not confuse the inequality $\lambda \gg L$, which is not required for the occurrence of superradiance in a sample of length $L$, with the necessary condition (17), which is compulsory for any coherence to develop in the system.

For each correlated region, the evolution equations (16) can be described in the singlemode picture and can be reduced to the guiding-center equations

$$
\begin{gathered}
\frac{d w}{d t}=-2 \gamma_{2}(1+\kappa-g s) w+2 \gamma_{3} s^{2}, \\
\frac{d s}{d t}=-g \gamma_{2} w-\gamma_{3} s-\gamma_{1}(s-\zeta),
\end{gathered}
$$

in which $\kappa \equiv \gamma_{2}^{*} / \gamma_{2}, \gamma_{3} \approx \gamma_{0}$ is the relaxation rate due to the local fluctuations caused by effective dipole-dipole interactions, and the coupling parameter

$$
g \equiv \rho \frac{\gamma_{0}}{\gamma_{2}} \int \frac{\sin k_{0}(r-z)}{k_{0} r} d \mathbf{r}
$$

describes the intensity of atomic interactions through the common radiation field. Under condition (17), the coupling parameter $g \sim N_{c} \gg 1$ is very large. For instance, for the $\mathrm{CO}_{2}$ laser with the atomic density $\rho \sim 10^{18} \mathrm{~cm}^{-3}$ and the working wavelength $\lambda \sim 10^{-3} \mathrm{~cm}$, estimating $N_{c}$ as $\rho \lambda^{3}$, we have $g \sim 10^{9}$. For solid state lasers, with $\rho \sim 10^{22}$ and $\lambda \sim 10^{-4}$, one has $g \sim 10^{10}$. Thus, condition (17) not only is compulsory for the development of coherence, but it is also favorable for having a large coupling parameter (20), which is necessary for achieving superradiance.

The development of atomic superradiance goes as follows. The system is prepared in a nonequilibrium state, with inverted population difference, so that $s_{0} \equiv s(0)>0$. No transverse fields are applied, so that $w_{0} \equiv w(0)=0$, since superradiance implies, by definition, a self-organized process. Atoms start with spontaneous emission, at the beginning being not correlated with each other. Their interactions through the radiated field yield the development of mutual correlations. After the crossover time $t_{c}=T_{2} / 2 g s_{0}$, these correlations become strong inside a correlated region of length $L_{c}$. Then the initial chaotic stage changes to the coherent stage, and atoms of each spatial mode start radiating coherently. The maximum of a superradiant burst happens at the delay time

$$
t_{0}=t_{c}+\frac{\tau_{p}}{2} \ln \left|\frac{2}{\gamma_{3} t_{c}}\right| .
$$

The duration of the superradiant pulse is given by the pulse time $\tau_{p}=T_{2} / g s_{0}$. Because of the very large coupling $g$, the pulse time can become an order shorter than $T_{2}$. The 
condition $\tau_{p} \ll T_{2}$ is the criterion for a coherent pulse to be called superradiant. Respectively, the intensity of radiation can be one or several orders stronger than that of incoherent radiation. In a concentrated system the intensity of a superradiant burst would be proportional to $N^{2}$, with $N$ being the total number of atoms. For realistic systems, with $\lambda \ll L$, where the radiation comes from several spatial modes, taking account of the spatial dispersion leads to the radiation intensity $I \sim N^{\alpha}$, with $1<\alpha<2$. The value of $\alpha$ depends on the sample shape. Thus for pencil-like or disk-like samples, one has $I \sim N^{5 / 3}$ or $I \sim N^{4 / 3}$, respectively [22].

It is important to stress that in an atomic system the relaxation rates $\gamma_{0}, \gamma_{2}$, and $\gamma_{3}$ all are caused by the same physical origin, by the radiation of atoms, and so all of them are of the same order of the natural width $\gamma_{0}$.

\section{Spin superradiance}

The microscopic description of spin superradiance has to be based on a realistic spin Hamiltonian [1,7]. For the generality of the consideration and in order to stress the principal difference from atomic systems, the spin Hamiltonian can be complimented by the field Hamiltonian $\hat{H}_{f}$, as in Eq. (3), and, respectively, by the spin interactions with electromagnetic field. Although electromagnetic spin interactions are negligible, as compared to direct dipole interactions [7], we shall keep them to demonstrate once again that they are not able to produce spin superradiance. This fact is what makes spin systems fundamentally different from atomic ones. To realize superradiance in a spin system, it is absolutely necessary to couple it to a resonant electric circuit $[1,7]$.

The system of $N$ spins is described by the Hamiltonian

$$
\hat{H}=\sum_{i=1}^{N} \hat{H}_{i}+\frac{1}{2} \sum_{i \neq j}^{N} \hat{H}_{i j}+\hat{H}_{f},
$$

where the single-spin term includes the interaction of spins with all magnetic fields, the second term is due to dipole spin interactions, and the last term is the field Hamiltonian (3). In the single-spin Hamiltonian

$$
\hat{H}_{i}=-\mu_{0} \mathbf{S}_{i} \cdot \mathbf{B}_{i}-D\left(S_{i}^{z}\right)^{2}
$$

$\mu_{0} \equiv \hbar \gamma_{S}$, with $\gamma_{S}$ being the gyromagnetic ratio of a particle with spin $S$. In what follows, we shall set $\hbar=1$. The Zeeman term of Eq. (22) includes the interaction of a spin $\mathbf{S}_{i}$ with the total magnetic field

$$
\mathbf{B}_{i}=B_{0} \mathbf{e}_{z}+H_{r e s} \mathbf{e}_{x}+\mathbf{H}_{i}
$$

where $B_{0}$ is a longitudinal external field, $H_{\text {res }}$ is a feedback field of a resonant electric circuit coupled to the sample, and $\mathbf{H}_{i} \equiv \mathbf{H}\left(\mathbf{r}_{i}, t\right)$ is the radiation field. The second term in Eq. (22) is the energy of the single-site magnetic anisotropy, with the anisotropy parameter $D$. The interaction Hamiltonian in Eq. (21) is due to direct dipole interactions

$$
\hat{H}_{i j}=\sum_{\alpha \beta} D_{i j}^{\alpha \beta} S_{i}^{\alpha} S_{j}^{\beta}
$$


with the dipolar tensor $D_{i j}^{\alpha \beta}$.

Writing down the Heisenberg equations of motion for spin operators $S_{i}^{\alpha} \equiv S^{\alpha}\left(\mathbf{r}_{i}, t\right)$, we shall average them over the spin degrees of freedom. Our aim is to obtain the evolution equations for the transverse function

$$
u(\mathbf{r}, t) \equiv \frac{1}{S}<S^{-}(\mathbf{r}, t)>
$$

coherence intensity

$$
w(\mathbf{r}, t) \equiv \frac{1}{S^{2}}<S^{+}(\mathbf{r}, t) S^{-}(\mathbf{r}+0, t)>,
$$

and the longitudinal spin polarization

$$
s(\mathbf{r}, t) \equiv \frac{1}{S}<S^{z}(\mathbf{r}, t)>
$$

These functions are analogous to Eqs. (7) to (9).

Let us introduce the notation for the effective force acting on a spin as

$$
f=f_{\text {res }}+f_{\text {rad }}+\xi \text {, }
$$

where the resonator force

$$
f_{\text {res }}=-i \mu_{0} H_{\text {res }}
$$

is caused by the action of the resonator feedback field $H_{\text {res }}$, the radiation force

$$
f_{\text {rad }}=\gamma_{r} u
$$

is due to spin interactions through the common radiation field [7,23], with the radiation rate

$$
\gamma_{r} \equiv \gamma_{0} N_{c}, \quad \gamma_{0} \equiv \frac{2}{3} \mu_{0}^{2} S k^{3}
$$

in which $N_{c}$ is the number of correlated spins and $\gamma_{0}$ is the natural radiation width; and the last term in Eq. (28) representing local spin fluctuations caused by dipole interactions $[1,7]$.

The effective transverse relaxation rate, owing its origin to dipole spin-spin interactions, can be represented [24] as

$$
\gamma_{2}(s)=\left(1-s^{2}\right) \gamma_{2}
$$

where

$$
\gamma_{2} \equiv \gamma_{2}(0)=n_{0} \rho \mu_{0}^{2} \sqrt{S(S+1)}
$$

with $n_{0}$ being the number of nearest neighbours and $\rho \equiv N / V$ being the spin density. Including also the inhomogeneous broadening $\gamma_{2}^{*}$, we have the total transverse relaxation rate

$$
\Gamma_{2}=\left(1-s^{2}\right) \gamma_{2}+\gamma_{2}^{*}
$$

Also, we shall employ the notation for the Zeeman frequency

$$
\omega_{0} \equiv-\mu_{0} B_{0}
$$


anisotropy frequency

$$
\omega_{D} \equiv(2 S-1) D,
$$

and the effective spin rotation frequency

$$
\omega_{s} \equiv \omega_{0}-\omega_{D} s
$$

Small frequency shifts, caused by the radiation friction and local dipole fluctuations, can be omitted [7]. Thus, we come to the equations

$$
\begin{gathered}
\frac{d u}{d t}=-i\left(\omega_{s}-i \Gamma_{2}\right) u+f s, \quad \frac{d w}{d t}=-2 \Gamma_{2} w+\left(u^{*} f+f^{*} u\right) s, \\
\frac{d s}{d t}=-\frac{1}{2}\left(u^{*} f+f^{*} u\right)-\gamma_{1}(s-\zeta),
\end{gathered}
$$

in which $\gamma_{1}$ is the spin-lattice attenuation and $\zeta$ is the value of a stationary spin polarization. These equations are analogous to Eqs. (16). Notice, however, that the frequency of spin rotation is $\omega_{s}$, given by Eq. (35), but not the Zeeman frequency (33).

In order that the interactions through electromagnetic radiation could lead to the appearance of noticeable correlations among spins, the correlated regions must exist of a typical length $L_{c}$, such that

$$
k L_{c} \ll 1 \quad\left(k \equiv \frac{\omega_{s}}{c}\right),
$$

which is in complete analogy with Eq. (17). Condition (37) is compulsory for the appearance of any coherence through the common radiation field. All related discussion, given above for atomic systems, is valid as well for spin systems. The correlation length $L_{c}$ can vary between the mean interspin distance $a$ and the linear system size $L$. Respectively, the number of correlated spins $N_{c}$ varies between $\rho a^{3}=1$ and the total number of spins $\rho L^{3}=N$.

Defining the resonator feedback field by the Kirchhoff equation, we find [1,7] the effective spin-resonator coupling

$$
g \equiv \frac{\gamma \bar{\gamma}_{0} \omega_{s}}{\gamma_{2}\left(\gamma^{2}+\Delta^{2}\right)}
$$

in which $\gamma$ is the circuit damping, the value

$$
\bar{\gamma}_{0} \equiv \pi \eta \rho \mu_{0}^{2} S,
$$

where $\eta$ is a filling factor, is of the order of $\gamma_{2}$ in Eq. (31), and $\Delta \equiv \omega-\left|\omega_{s}\right|$ is a detuning between the circuit natural frequency $\omega$ and the spin rotation frequency $\omega_{s}$, given by Eq. (35). Taking into account that $\gamma \equiv \omega / 2 Q$, where $Q$ is a resonator quality factor, we have $g \sim Q$, which can be as large as $g \sim 10^{3}-10^{5}$.

The effective spin coupling through the radiation field is

$$
g_{r} \equiv \frac{\gamma_{r}}{\gamma_{2}}=\frac{2}{3 n_{0}} \sqrt{\frac{S}{S+1}}\left(k L_{c}\right)^{3} .
$$


According to the necessary condition (37), and since $n_{0} \sim 10$, we get $g_{r} \ll 1$.

Solving the first of equations (36) with respect to $u$ and substituting this solution to the second and third of Eqs. (36), we obtain, after the averaging procedure over fast oscillations $[1,7]$, the guiding-center equations

$$
\begin{gathered}
\frac{d w}{d t}=-2 \gamma_{2}\left(1+\kappa-s^{2}-g s-g_{r} s\right) w+2 \gamma_{3} s^{2}, \\
\frac{d s}{d t}=-\gamma_{2}\left(g+g_{r}\right) w-\gamma_{3} s-\gamma_{1}(s-\zeta),
\end{gathered}
$$

in which $\kappa \equiv \gamma_{2}^{*} / \gamma_{2}$ and $\gamma_{3} \leq \gamma_{2}$ is an attenuation owing its origin to the local spin fluctuations related to dipole interactions. These are the evolution equations that are to be compared with Eqs. (18) and (19).

Because of a negligibly small value of the radiation coupling $g_{r} \ll 1$, it plays no role in spin dynamics. It is easy to check that even when there is no resonator coupling, that is $g \equiv 0$, the terms with $g_{r} \ll 1$ do not influence the spin motion described by Eqs. (40). This implies that the interaction of spins through the common radiation field can never produce spin superradiance. This conclusion is general and is valid for any type of spins, whether these are nuclear, electron, or molecular spins. Contrary to the negligible radiation coupling (39), the spin-resonator coupling (38), as is explained above, can be made very large. It is only by coupling a spin sample to a resonant electric circuit that one is able to realize spin superradiance $[1,7]$. This drastic difference of spin systems from atomic ones comes from the fact that in the former the linewidth $\gamma_{2}$ and the radiation rate $\gamma_{r}$ are caused by distinct physical origins, while in atomic systems they are of the same origin. In spin systems, the radiation rate $\gamma_{r} \ll \gamma_{2}$ is negligible, as compared to the dipole linewidth $\gamma_{2}$. On the contrary, in atomic systems the collective radiation rate $g \gamma_{2} \gg \gamma_{2}$ is much larger than the linewidth $\gamma_{2}$.

In a spin sample coupled to a resonant circuit, spin superradiance takes place in the following way. The sample is prepared in an inverted state, when its average magnetization is opposite to an external magnetic field. Initial spin motion is triggered by local spin fluctuations caused by dipole interactions. Chaotic fluctuations last till the chaos time $t_{c} \approx \tau / g s_{0}$, where $\tau \equiv \gamma^{-1}$ is the resonator ringing time and $s_{0} \equiv s(0)$. Then correlations in the transverse spin motion begin rising fast, being due to the collectivization through the resonator feedback field. The superradiant burst peaks at the delay time

$$
t_{0}=t_{c}+\frac{\tau_{p}}{2} \ln \left|\frac{2}{\gamma_{3} t_{c}}\right| .
$$

The pulse time $\tau_{p}=T_{2} / g s_{0}$ is much shorter than $T_{2}$, which is necessary for a pulse to be treated as superradiant.

The possibility of superradiant operation by spin systems can be used in a number of applications [1,7], for example, by creating spin masers [14]. By analogy with squeezed atomic states [25], one can realize spin squeezing. Punctuated spin superradiance can be employed for information processing [26].

In recent years, much attention is given to the consideration of molecular magnets consisting of molecules whose spin can reach high values. The feasibility of realizing the resonator-assisted spin superradiance in such materials was advanced in Ref. [14]. At 
the same time, there have appeared speculations $[13,27]$ that superradiance in molecular magnets could occur due only to spin interactions through the common radiation field. As is thoroughly explained above, this is certainly impossible. Spin superradiance can happen only as a resonator-assisted phenomenon [14]. There have been attempts of measuring possible microwave emission generated in the avalanches of magnetization reversal in $\mathrm{Mn}_{12}$-acetate at low temperatures [28]. However in other highly precise experiments [29] no significant radiation at well-defined frequencies was detected. Actually, the lowtemperature step-like avalanches in $\mathrm{Mn}_{12}$ acetate are known for a long time and can be due to different reasons [30]. But, as the accurate theory shows, these avalanches cannot be related to superradiance.

Moreover, in a typical experimental setup for such a molecular magnet as $\mathrm{Mn}_{12}$-acetate, the process of an avalanche is not characterized by a well-defined frequency. Really, let us consider the values of the parameters for $\mathrm{Mn}_{12}$-acetate, whose spin is $S=10$. Then $D \cong 0.6 \mathrm{~K}$, so that in frequency units $D=0.8 \times 10^{11} \mathrm{~s}^{-1}$, and the anisotropy frequency (34) is $\omega_{D}=1.5 \times 10^{12} \mathrm{~s}^{-1}$. The density is $\rho=2.2 \times 10^{20} \mathrm{~cm}^{-3}$. Using $\gamma_{S}=-1.759 \times 10^{7}$ $\mathrm{G}^{-1} \mathrm{~s}^{-1}, \mu_{0}=-1.856 \times 10^{-20} \mathrm{erg} / \mathrm{G}$, and $\mu_{0} \gamma_{S}=3.265 \times 10^{-13} \mathrm{~cm}^{3} / \mathrm{s}$, we find the dipole linewidth (31) as $\gamma_{2}=0.8 \times 10^{10} \mathrm{~s}^{-1}$. The Zeeman frequency (33) for the field $B_{0}=1 T=10^{4} \mathrm{G}$ is $\omega_{0}=1.76 \times 10^{11} \mathrm{~s}^{-1}$, for $B_{0}=2 T$, one has $\omega_{0}=3.52 \times 10^{11} \mathrm{~s}^{-1}$, and $\omega_{0}=5.28 \times 10^{11} \mathrm{~s}^{-1}$ for $B_{0}=3 T$. Experiments with magnetization avalanches are usually done in the field of $2-3 T$. For such fields, the Zeeman frequency $\omega_{0} \sim 10^{11} \mathrm{~s}^{-1}$ is much smaller than the anisotropy frequency $\omega_{D} \sim 10^{12} \mathrm{~s}^{-1}$. Hence, the spin rotation frequency (35) is defined mainly by the term $-\omega_{D} s$. In the process of an avalanche, the spin polarization $s$ changes from $s=1$ to $s=-1$. The spin frequency varies by the amount $\Delta \omega_{s}=-\omega_{D} \Delta s$. For the polarization change $\Delta s=-2$, the frequency variation is $\Delta \omega_{s}=2 \omega_{D}=3 \times 10^{12} \mathrm{~s}^{-1}$. Even if $s$ changes by $\Delta s=-0.1$, corresponding to one quantum transition, the spin frequency varies as $\Delta \omega_{s}=0.1 \omega_{D}=1.5 \times 10^{11} \mathrm{~s}^{-1}$. This means that there is no well-defined radiation frequency during the avalanche.

Recall that the appearance of the radiation rate (29) as such is preconditioned by the existence of a well-defined constant radiation frequency. If the latter does not exist, then the radiation rate (29) has no sense, as far as no coherent radiation can arise in principle.

Concluding, spin superradiance can be realized in spin systems only by coupling them to a resonant electric circuit. To preserve the stability of the spin frequency $\omega_{s}$, one can either impose a sufficiently strong external magnetic field $B_{0}$, such that $\omega_{0}$ be essentially larger than $\omega_{D}$, or by varying $B_{0}$ to achieve the chirping effect, as described in Refs. [7,14].

Acknowledgements. I am grateful to E.P. Yukalova for useful discussions. The Mercator Professorship of the German Research Foundation is appreciated. 


\section{References}

[1] V.I. Yukalov, in: Encyclopedia of Nuclear Magnetic Resonance, edited by D.M. Grant and R.K. Harris (Wiley, Chichester, 2002), Vol. 9, p. 697.

[2] R.H. Dicke, Phys. Rev. 93, 99 (1954).

[3] A.V. Andreev, V.I. Emelyanov, and Y.A. Ilinskii, Cooperative Effects in Optics (Institute of Physics, Bristol, 1993).

[4] L. Mandel and E. Wolf, Optical Coherence and Quantum Optics (Cambridge University, Cambridge, 1995).

[5] J.F. Kiselev, A.F. Prudkoglyad, A.S. Shumovsky, and V.I. Yukalov, Mod. Phys. Lett. B 1, 409 (1988).

[6] Y.F. Kiselev, A.F. Prudkoglyad, A.S. Shumovsky, and V.I. Yukalov, J. Exp. Theor. Phys. 67, 413 (1988).

[7] V.I. Yukalov and E.P. Yukalova, Phys. Part. Nucl. 35, 348 (2004).

[8] V.I. Yukalov, Phys. Rev. Lett. 75, 3000 (1995).

[9] V.I. Yukalov, Laser Phys. 5, 526 (1995).

[10] V.I. Yukalov, Laser Phys. 5, 970 (1995).

[11] V.I. Yukalov, Phys. Rev. B 53, 9232 (1996).

[12] V.I. Yukalov, Laser Phys. 7, 58 (1997).

[13] E.M. Chudnovsky and D.A. Garanin, e-print cond-mat/0406621 (2004).

[14] V.I. Yukalov, Laser Phys. 12, 1089 (2002).

[15] V.I. Yukalov, Laser Phys. 14, 1403 (2004).

[16] V.I. Yukalov and E.P. Yukalova, Phys. Part. Nucl. 31, 561 (2000).

[17] V.I. Yukalov, in: Mathematical Physics, edited by L.D. Faddeev (Grand Russian Encyclopedia, Moscow, 1998), p. 169.

[18] V.I. Yukalov, J. Mod. Opt. 35, 35 (1988).

[19] V.I. Yukalov, J. Mod. Opt. 37, 1361 (1990).

[20] V.I. Yukalov, Phys. Lett. A 278, 30 (2000).

[21] V.I. Yukalov, Phys. Lett. A 284, 91 (2001).

[22] V.I. Yukalov, Eur. Phys. J. D 13, 83 (2001).

[23] V.L. Ginzburg, J. Exp. Theor. Phys. 13, 33 (1943). 
[24] A. Abragam and M. Goldman, Nuclear Magnetism: Order and Disorder (Clarendon, Oxford, 1982).

[25] V.V. Dodonov. J. Opt. B 4, R1 (2002).

[26] V.I. Yukalov and E.P. Yukalova, Phys. Rev. Lett. 88, 257601 (2002).

[27] C.L. Joseph, C. Calero, and E.M. Chudnovsky, e-print cond-mat/0406361 (2004).

[28] A. Hernandez-Mínguez, M. Jordi, R. Amigo et al., e-print cond-mat/0406389 (2004).

[29] M. Bal, J.R. Friedman, K. Mertez et al., e-print cond-mat/0406410 (2004).

[30] C. Paulsen, J.G. Park, B. Barbara et al., J. Magn. Magn. Mater. 140, 1891 (1995). 